\title{
On the mechanism of trailing vortex wandering
}

\author{
Adam M. Edstrand ${ }^{1} \dagger$, Timothy B. Davis ${ }^{1}$, Peter J. Schmid ${ }^{2}$, \\ Kunihiko Taira ${ }^{1}$, and Louis N. Cattafesta III $^{1}$ \\ ${ }^{1}$ Department of Mechanical Engineering, Florida Center for Advanced Aero-Propulsion, \\ 2003 Levy Ave., Florida State University, Tallahassee, FL 32310, U.S.A. \\ ${ }^{2}$ Department of Mathematics, Imperial College London, London SW7 2AZ, United Kingdom
}

(Received $\mathrm{xx}$; revised $\mathrm{xx}$; accepted $\mathrm{xx}$ )

The mechanism of trailing vortex wandering has long been debated and often attributed to either wind-tunnel effects or a self-induced instability. We remove the effect of wandering from a measured velocity field by averaging and, through a triple decomposition, recover the coherent wandering motion. Based on this wandering motion, the most energetic structures are computed using the proper orthogonal decomposition (POD) and exhibit a helical mode $|m|=1$ whose kinetic energy grows with downstream progression. As such, we hypothesize that a vortex instability underlies the wandering motion, and test this hypothesis by performing a spatial stability analysis of a matched Batchelor vortex, which is devoid of wind-tunnel effects. The primary stability mode is marginally stable and is nearly identical, in size and structure, to the principal POD mode. The strikingly similar structure coupled with the measured energy growth supports the proposition that the vortex wandering is the result of an instability. The cause of the wandering is the non-zero radial velocity of the $|m|=1$-mode on the vortex centerline, transversely displacing the trailing vortex as observed in experiments. However, the marginal nature of the stability mode prevents any conclusion regarding the specific type of instability.

\section{Background}

In the 1970s, researchers observed "vortex wandering" (also referred to as meandering) in wind-tunnel experiments that, through coherent side-to-side motion, effectively concealed pertinent characteristics of the measured core of the trailing vortex (Corsiglia et al. 1973; Baker et al. 1974). Although this phenomenon has been observed in experiments for decades, the underlying mechanism causing the wandering remains uncertain. Despite this, the effects of wandering can be corrected (Devenport et al. 1996); most pertinent for the present study is the method by Heyes et al. (2004), which corrects for wandering directly using particle image velocimetry (PIV). This method shifts each instantaneous vortex image to collocate with the mean vortex center position, directly removing the effect of wandering, while retaining the instantaneous velocity field and its random fluctuations within the vortex core.

Many researchers speculate that wandering is a consequence of either wind-tunnel effects (Corsiglia et al. 1973; Devenport et al. 1996; Beresh et al. 2010) or a self-induced vortex instability (Rokhsaz et al. 2000; Jacquin et al. 2001). Wandering persists at zero angle of attack, indicating that it occurs despite the presence of a vortex (Baker et al. 1974), which may suggest that the motion is independent of the vortex itself and may be a result of the wind-tunnel environment. Conversely, there is an increase in the level of

$\dagger$ Email address for correspondence: aedstrand@fsu.edu 
the vortex wandering with downstream position (Devenport et al. 1996), potentially indicating an instability mechanism. Moreover, Jacquin et al. (2001) found that wandering is unaffected by the wind tunnel walls, while the frequencies of vortex instabilities found in literature match well with the frequencies of the wandering. Through a stochastic forcing of a Lamb-Oseen vortex, Fontane et al. (2008) found a long-wavelength helical mode to cause "global translation" of the vortex consistent with wandering. Despite the arguments on each side, correcting for wandering without certainty of its origin is precarious as one may disregard pertinent features of the flow field.

The purpose of the present study is to further examine the hypothesis that vortex wandering is due to an inherent instability. Using stereoscopic particle image velocimetry (SPIV) data (Deem et al. 2013), a new approach is developed to extract the coherent wandering motion. This motion is solved through a triple decomposition (Reynolds \& Hussain 1972) of the SPIV data, and the most energetic structures are determined through a proper orthogonal decomposition (Sirovich 1987). We then fit a canonical Batchelor vortex to the mean experimental data, and perform a spatial stability analysis on the resulting base flow field. The results show a strikingly similar spatial structure between the principal POD mode and the primary stability mode of the Batchelor vortex. Based on this similarity, coupled with the measured growth in kinetic energy, we propose wandering as a vortex instability rather than an artifact of the wind-tunnel environment.

\section{Methodology}

\subsection{Experimental setup}

The trailing vortex was generated by a $304.8 \mathrm{~mm}$ NACA0012 airfoil with a half-aspect ratio of 1.25 , a rectangular wingtip, and positioned at an angle of attack of $5^{\circ}$. All measurements, non-dimensionalized by freestream velocity and chord, were acquired at $R e_{c}=530,000$ in the University of Florida Anechoic Flow Facility (Mathew et al. 2005), a $1.12 \mathrm{~m} \times 0.74 \mathrm{~m}$ open-jet wind tunnel installed inside a semi-anechoic chamber. Owing to the open-jet nature of the facility, the wandering effects may be further exacerbated by the shear layers on three of the four sides, when compared to conventional wind tunnels with rigid tunnel walls. However, we expect minimal differences between open and closedwall settings as the wind-tunnel blockage is less than $1.5 \%$ with a thin streamlined body at a small angle of attack. We performed SPIV measurements perpendicular to the free stream from $x / c=0.67$ to 5 on eight measurement planes as shown in figure 1 . These measurements focused on the vortex core, which contained the two-dimensional wake of the wing up to $x / c \approx 3$, after which the wake exited the measurement plane due to downwash. Details of the experimental setup are provided in Deem et al. (2013). The instantaneous vortex centers, as computed by the method of Heyes et al. (2004) and shown in figure 2(b), and the fit Gaussian joint probability density function (jpdf), shown in figure $2(a)$, demonstrate that wandering is present and significant, with a slight correlation between the $y$ - and $z$-motion. After removing the wandering motion, the mean flow field is shown in $2(c)$.

\subsection{Triple decomposition}

A triple decomposition (Reynolds \& Hussain 1972) extracts the coherent wandering motion from the SPIV data. To this end, for each SPIV snapshot, the vortex centers are collocated by linearly shifting the instantaneous core positions to the mean vortex axis, shown as $\times$ in figure $2(b)$. This results with the corrected instantaneous flow field $\hat{\boldsymbol{u}}(\boldsymbol{x}-$ $\left.\boldsymbol{x}_{\boldsymbol{c}}\left(t_{k}\right), t_{k}\right)$, where the image is shifted by $\boldsymbol{x}_{\boldsymbol{c}}\left(t_{k}\right)$. The corrected mean flow field $\overline{\hat{\boldsymbol{u}}}$ is then 


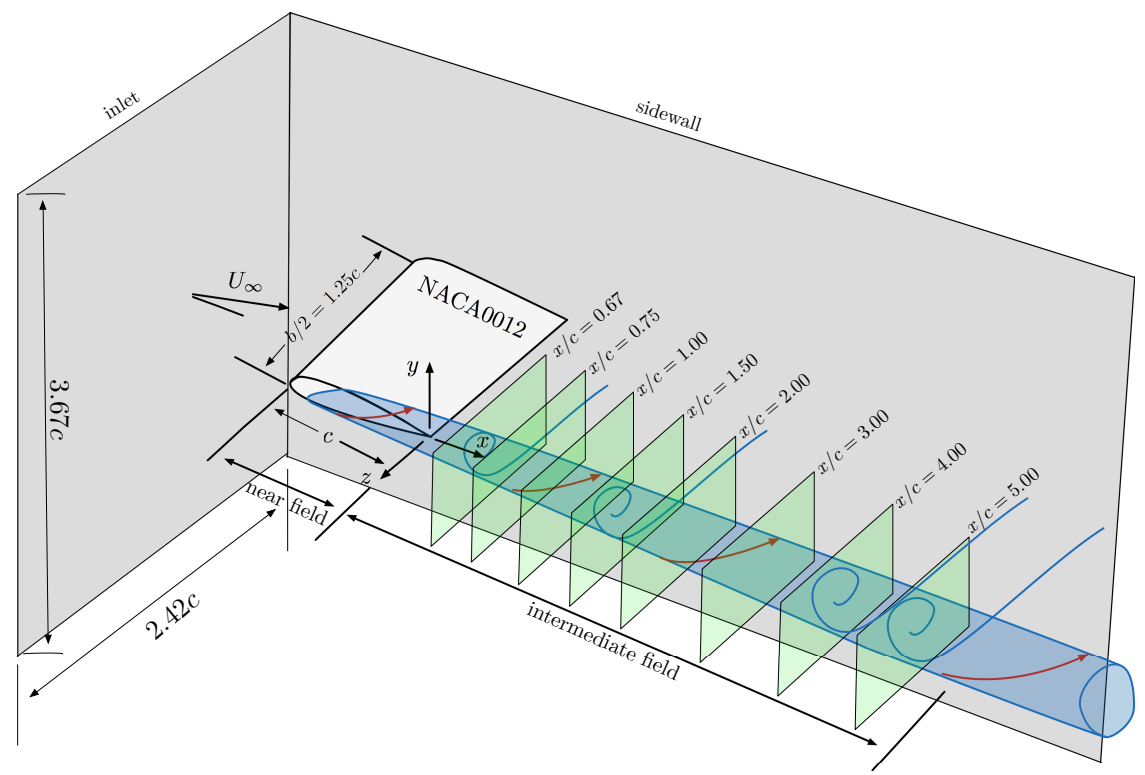

Figure 1: Schematic of the trailing vortex and coordinate system. The blue cone denotes the area of the vortex core, while the green planes represent the areas of available stereoscopic particle image velocimetry data. The wing chord is $c=304.8 \mathrm{~mm}$.

(a)

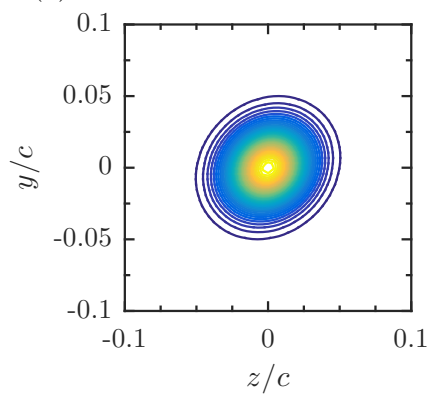

(b)

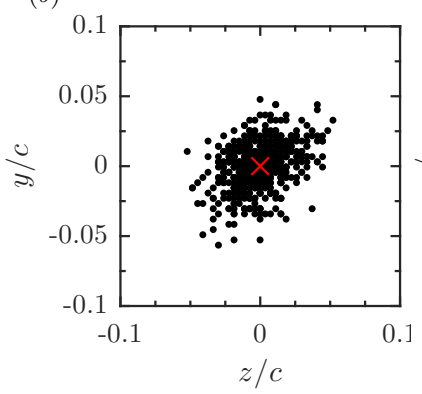

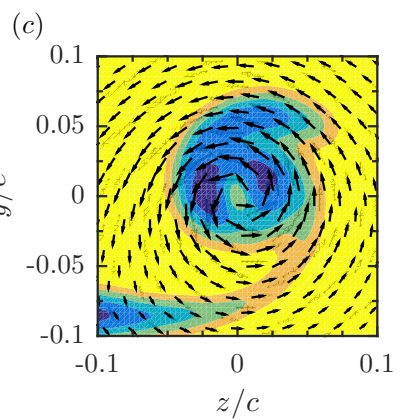

Figure 2: The joint probability density function (jpdf) of the vortex center location at $x / c=5$. (a) the Gaussian jpdf with the parameters from the experimentally obtained vortex centers, shown in $(b)$. The red $\times$ in $(b)$ denotes the mean vortex location, and the computed correlation coefficient is $\rho=0.24$. The mean flow field at $x / c=0.67$ is shown in $(c)$. The contours of $U / U_{\infty}$ are displayed between 0.9 and 1 , and the quiver plot shows the transverse and normal velocities.

obtained by averaging the snapshots in time according to

$$
\overline{\hat{\boldsymbol{u}}}(\boldsymbol{x})=\frac{1}{N} \sum_{k=1}^{N} \hat{\boldsymbol{u}}\left(\boldsymbol{x}-\boldsymbol{x}_{\boldsymbol{c}}\left(t_{k}\right), t_{k}\right) .
$$


We obtain the velocity fluctuations devoid of wandering, $\boldsymbol{u}^{\prime}$, for each snapshot by subtracting the mean via

$$
\boldsymbol{u}^{\prime}\left(\boldsymbol{x}, t_{k}\right)=\hat{\boldsymbol{u}}\left(\boldsymbol{x}-\boldsymbol{x}_{\boldsymbol{c}}\left(t_{k}\right), t_{k}\right)-\overline{\hat{\boldsymbol{u}}}(\boldsymbol{x}) .
$$

We then assume vortex wandering to be the consequence of a coherent motion, and by using the triple decomposition of Reynolds \& Hussain (1972), the instantaneous flow field is decomposed into mean, coherent, and random components, given by

$$
\boldsymbol{u}\left(\boldsymbol{x}, t_{k}\right)=\overline{\boldsymbol{u}}(\boldsymbol{x})+\tilde{\boldsymbol{u}}\left(\boldsymbol{x}, t_{k}\right)+\boldsymbol{u}^{\prime}\left(\boldsymbol{x}, t_{k}\right) .
$$

For the SPIV data, $\boldsymbol{u}$ represents the instantaneous measured velocity field, $\overline{\boldsymbol{u}}$ denotes the time-averaged velocity field without wandering correction, $\tilde{\boldsymbol{u}}$ is the coherent wandering velocity field, and $\boldsymbol{u}^{\prime}$ is the random perturbation obtained from equation (2.2). It is straightforward to solve for $\tilde{\boldsymbol{u}}$ using equations (2.3) and (2.2) to obtain

$$
\tilde{\boldsymbol{u}}\left(\boldsymbol{x}, t_{k}\right)=\left[\boldsymbol{u}\left(\boldsymbol{x}, t_{k}\right)-\overline{\boldsymbol{u}}(\boldsymbol{x})\right]-\left[\hat{\boldsymbol{u}}\left(\boldsymbol{x}, t_{k}\right)-\overline{\hat{\boldsymbol{u}}}(\boldsymbol{x})\right],
$$

where the first bracketed term contains the coherent wandering motion and background turbulence, while the second bracketed term removes the turbulence. The resulting velocity field, $\tilde{\boldsymbol{u}}\left(\boldsymbol{x}, t_{k}\right)$, describes the coherent wandering motion. From these snapshots, the most energetic structures of the coherent portion are extracted via the POD (Sirovich 1987).

\subsection{Batchelor vortex model}

For comparison of the experimental results with a canonical vortex base flow, the Batchelor vortex (Batchelor 1964) is fit to the extracted mean flow,

$$
V_{x}=1-\gamma \exp \left(-\frac{r^{2}}{\delta^{2}}\right), \quad V_{r}=0, \quad V_{\theta}=\frac{\kappa}{r}\left[1-\exp \left(-\frac{r^{2}}{\delta^{2}}\right)\right],
$$

originally developed as a model for trailing vortices. In the above expression, $\kappa$ represents the swirl parameter, $\delta$ is the core radius, and $\gamma$ denotes the axial velocity deficit at $r=0$. All these parameters are non-dimensionalized by the free-stream velocity, $U_{\infty}^{*}$ and the airfoil chord, $c$. To fit equation 2.5 to the experimental data, the mean velocity field, $\overline{\boldsymbol{u}}$, is azimuthally averaged (see figure $3(c)$ ) and curve-fit using a nonlinear least squares method. Shown in figure $3(a)-(b)$ is the resulting fit with the non-dimensional parameters $\kappa=0.0152, \delta=\delta^{*} / c=0.0514$, and $\gamma=0.0671$, non-dimensionalized by the chord, $c=0.3048 \mathrm{~m}$ and $U_{\infty}=28.5 \mathrm{~m} / \mathrm{s}$. These values corresponds to the non-dimensional swirl parameter of $q=\kappa /(\delta \gamma)=4.39$ of a $q$-vortex. These parameters corresponds to a Reynolds number based on the core radius, of $R e_{\delta^{*}}=R e_{c} \delta=27,200$. Averaging over the azimuth, excluding the two-dimensional wake, shows that the fitting parameters are rather insensitive to the averaging region; the swirl $\kappa$ has been found to be the most sensitive parameter, varying by $11.5 \%$.

\subsection{Linear stability analysis}

The governing equations for infinitesimal disturbances are given by

$$
\begin{gathered}
\nabla \cdot \tilde{\boldsymbol{u}}=0 \\
\frac{\partial \tilde{\boldsymbol{u}}}{\partial t}+\boldsymbol{U} \cdot \nabla \tilde{\boldsymbol{u}}+\tilde{\boldsymbol{u}} \cdot \nabla \boldsymbol{U}=-\nabla \tilde{p}+\frac{1}{R e_{\delta}} \nabla^{2} \tilde{\boldsymbol{u}}
\end{gathered}
$$

where $\tilde{\boldsymbol{u}}=\left[\tilde{v}_{x}, \tilde{v}_{r}, \tilde{v}_{\theta}\right]^{T}$ denotes the disturbance velocity field, $\boldsymbol{U}=\left[V_{x}, V_{r}, V_{\theta}\right]^{T}$ is the base velocity field, and $\tilde{p}$ represents the disturbance pressure. The experimentally 

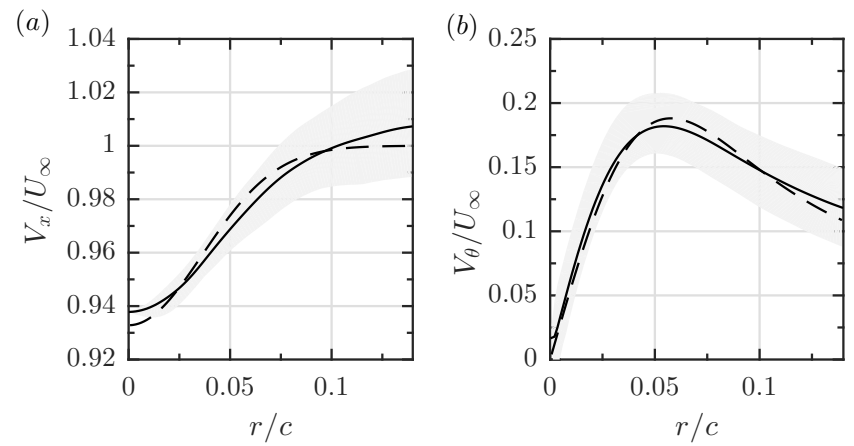

$(c)$

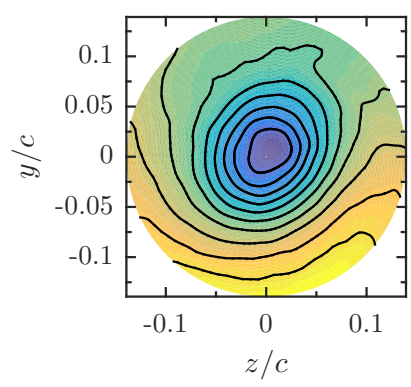

Figure 3: Velocity distributions from experimental data for the $(a)$ streamwise velocity and the $(b)$ azimuthal velocity. The azimuthal average of the velocities are shown by the solid line, with the shaded area representing the variations. Using this mean flow field, the Batchelor vortex is fit using a least-squares method, shown by the dashed line. The contours in $(c)$ show the region of azimuthal averaging for the Batchelor fit. For this fit, the parameters are found to be $\kappa=0.0152, \delta=0.0514$, and $\gamma=0.0671$, which corresponds to a swirl parameter of $q=4.39$.

measured vortex as well as the Batchelor vortex vary slowly with downstream progression, making streamwise variations higher-order in nature. As such, we employ the parallel approximation and assume wave-like disturbances of the form $\tilde{\boldsymbol{q}}(x, r, \theta, t)=$ $\boldsymbol{q}(r) \exp (i \alpha x+i m \theta-i \omega t)$, while neglecting streamwise gradients in the base flow. Here, $\alpha \in \mathbb{C}$ is the streamwise wavenumber, $m$ is the azimuthal wavenumber, and $\omega \in \mathbb{R}$ is the radian frequency. We perform a spatial stability analysis, resulting in the generalized eigenvalue problem of the form

$$
\mathcal{A} \boldsymbol{q}_{*}=\alpha \mathcal{B} \boldsymbol{q}_{*},
$$

where, $\boldsymbol{q}_{*}=\left[v_{x}, v_{r}, v_{\theta}, p, \alpha v_{r}, \alpha v_{\theta}\right]^{T}$ are the shape functions of the perturbation, while $\mathcal{A}$ and $\mathcal{B}$ are the resulting stability matrices, omitted for brevity (see, e.g., Paredes (2014)). The coefficient matrices $\mathcal{A}$ and $\mathcal{B}$ are discretized using a Chebyshev spectral collocation method and treatment of the singularity is handled through the method of Mohseni \& Colonius (2000). The disturbance is evaluated on a mapped Gauss-Lobatto grid for $r / \delta \in[0,100]$ (Hein \& Theofilis 2004), where Dirichlet boundary conditions are enforced at the computational boundary, forcing the disturbances to zero in the far field. The current formulation has been validated with the Batchelor vortex results of Paredes (2014), and the variation of the fitting parameters, $\kappa, \delta$, and $\gamma$ affected the growth rates of the stability analysis by $2.4 \%$.

\section{Results}

Figure 4 illustrates the principal POD mode as it progresses downstream. Upstream of $x / c=1$, the streamwise velocity (first column) demonstrates how the eventual helical mode forms in the presence of the two-dimensional wake. The existence of the wake implies a coupling between the vortex and wake mode, underlining that, at the upstream locations, both the two-dimensional wake and vortex are necessary to accurately model these modes. With downstream progression, however, the downwash from the vortex separates the two-dimensional wake from the vortex, consequently 
diminishing the coupling and isolating the vortex, which in turn develops the classical helical pattern.

The middle and right columns of figure 4 show the transverse velocities. An important characteristic is that these velocities support a non-zero magnitude at the vortex centerline, which perturbs the vortex core by transversely displacing it and thus causing a wandering motion. This particular mode rotates in time; however, the direction of rotation remains undetermined, because the data are insufficiently resolved in time. Therefore, we simply denote their azimuthal wavenumber by its magnitude as $|m|=1$. The two-dimensional wake is absent in these modes, indicating that the small transverse velocity gradients in the wake are insignificant. The structure of these velocities are maintained with downstream progression; only growth as a consequence of viscous diffusion of the trailing-vortex flow field is observed.

Figure $5(a)$ shows the kinetic energy of the first four modes as they progress downstream. All modes monotonically increase, with no mode-switching between them. The dominant two modes are paired helical $|m|=1$ modes, while the lower energy modes consist of higher-order azimuthal modes (e.g., $|m|=2$ ). The monotonic growth implies that the wandering mode is dominant and displays similarities to the monotonic growth of the wandering amplitude shown in previous studies (Devenport et al. 1996). Figure 5(b) shows that the overall coherent kinetic energy linearly grows in the downstream direction. The observed energy growth coupled with the helical mode supports our proposition that the wandering motion is the consequence of a vortex instability.

The decoupling of the wake and the vortex in the transverse velocities suggest that the wake only insignificantly affects the transverse motion, thus implying that the wake is unnecessary to model the base-flow for the wandering motion. As a result, we fit a Batchelor-vortex profile to the mean flow field, as discussed in $\S 2.3$, at the $x / c=5$ location where any wake component is absent from even the streamwise velocity component. This procedure provides a base flow for our stability analysis. As we observe energy growth in the streamwise direction, we conduct a spatial stability analysis on the matched Batchelor vortex by prescribing a wandering frequency $\omega$. A frequency sweep, shown in figure $6(a)$, yields the most unstable eigenvalue $\alpha=0.5420-i 3.67 \times 10^{-6}$ at a frequency of $\omega=0.56$. The corresponding dimensional frequency of the least stable mode is given as $f^{*}=\omega U_{\infty} /(2 \pi c)=8.2 \mathrm{~Hz}$, which aligns well with the frequencies of vortex wandering reported by Devenport et al. (1996) and Bailey \& Tavoularis (2008).

At this frequency, figure $6(b)$ presents the spatial eigenvalue spectrum, which yields a single eigenvalue in the discrete branch near the neutral line, $\alpha_{i}=0$. The remaining eigenvalues fall on the continuous branch; the corresponding eigenfunctions describe freestream oscillations that decay in the streamwise coordinate direction. Therefore, the marginally stable mode, denoted by the red $\times$, would prevail far downstream, as all other modes decay to zero.

The eigenfunctions of this $m=-1$ marginally stable mode are shown in figure $7(a)-(c)$, and compared to the POD mode at the equivalent $x / c=5$ position (figure $7(d)-(f)$ ). The POD and stability modes demonstrate striking similarities, showing nearly identical size and structure with one another. The stability mode is characterized by a two-lobed helical structure in the streamwise velocity, with non-zero transverse velocity at the vortex core. The structure of this mode proves to be robust to variations in the base flow, which testifies to the resilience of this mode seen across a variety of experimental conditions over many years of study.

Regardless of the inconsistency between growth rates, the matching structure from the stability and the POD-analyses, together with the measured energy growth in the streamwise direction, strongly points towards an instability as the underlying mechanism 
(a)

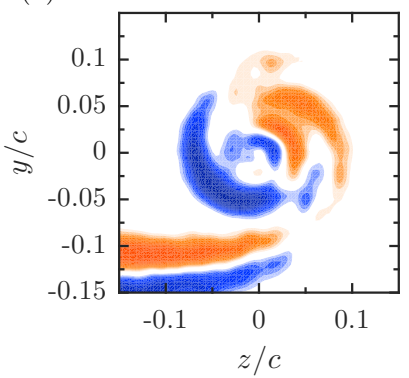

(d)

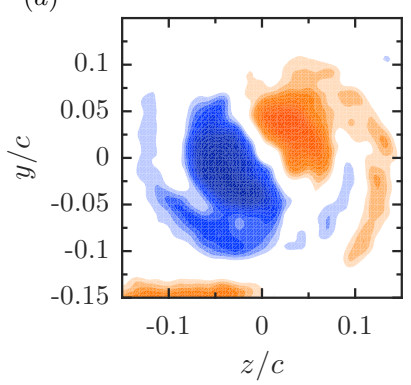

(g)

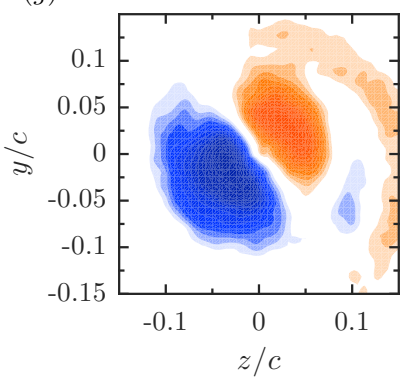

(j)

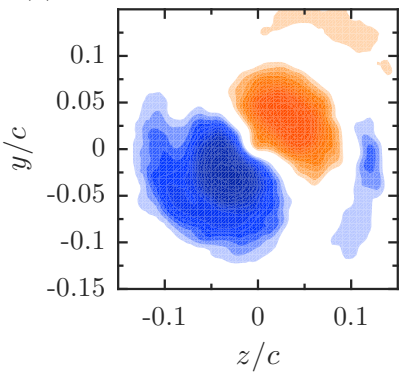

(b)

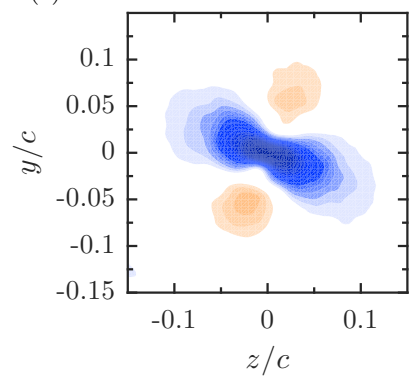

(e)

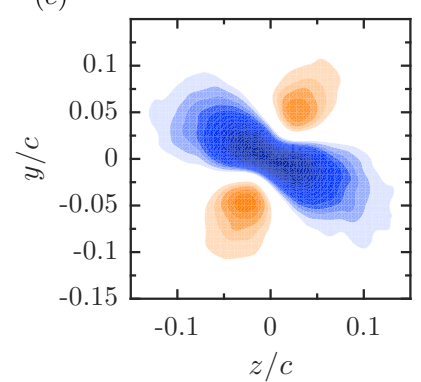

(h)

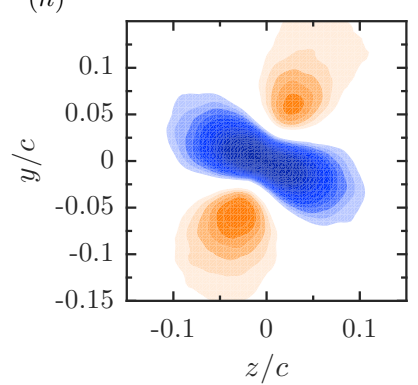

(k)

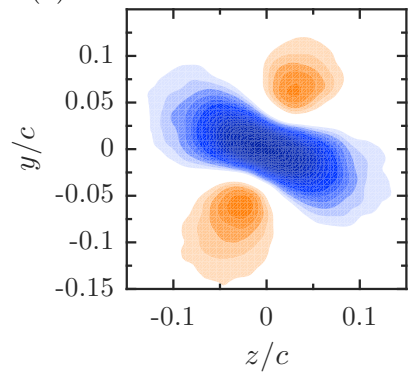

(c)

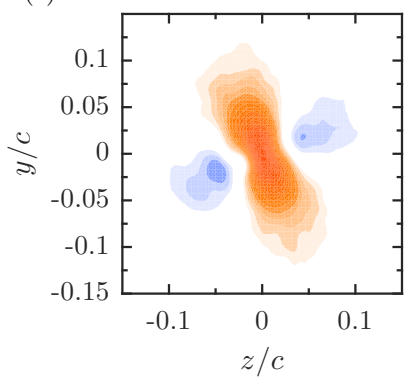

(f)

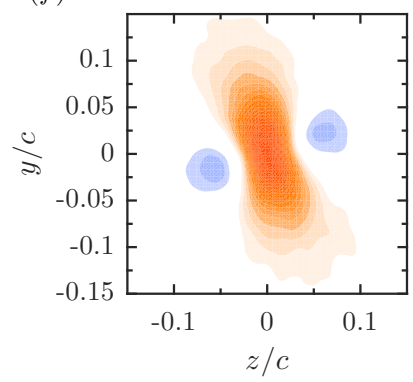

(i)

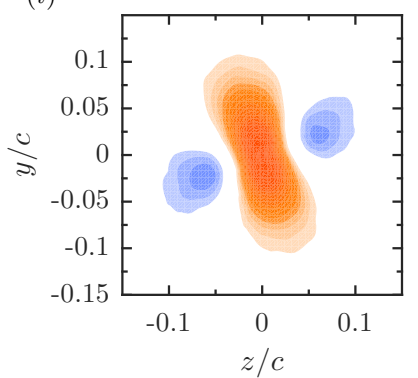

(l)

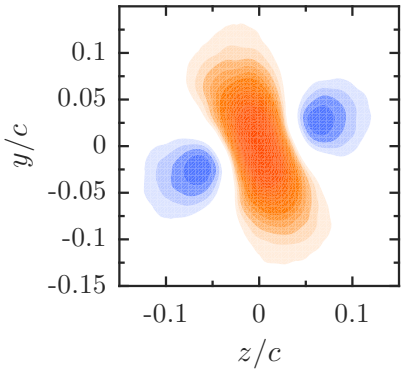

Figure 4: Principal POD mode for $\tilde{u}, \tilde{v}, \tilde{w}$ shown in the left, middle and right columns, respectively. From top to bottom, the rows correspond to the downstream distances $x / c=1,2,3$, and 4 . 
(a)

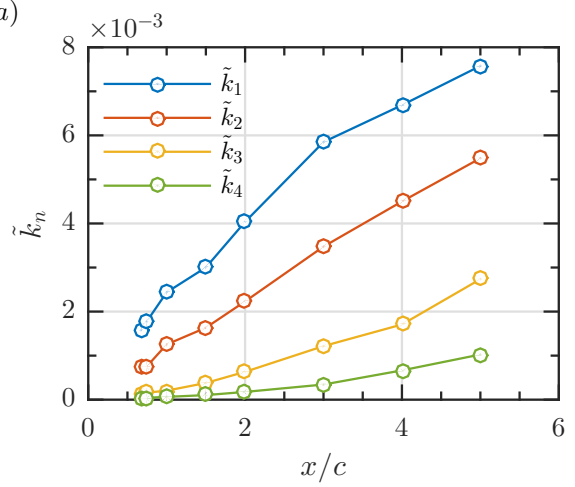

(b)

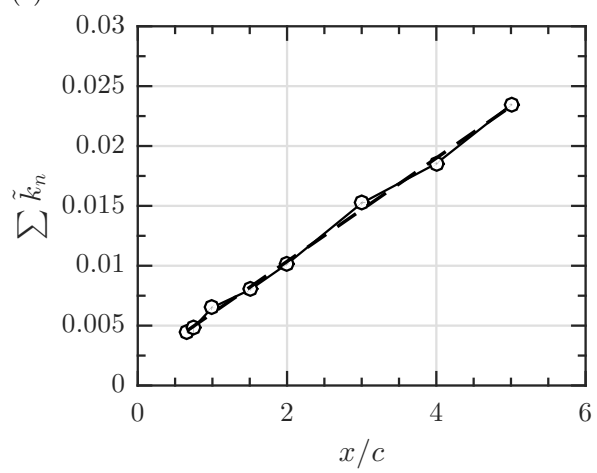

Figure 5: Kinetic energy, $\tilde{k}_{n}$, of POD modes $n=1,2,3$, and 4 in $(a)$. The coherent kinetic energy, $(b)$ representing the sum of all the individual modes with a linear fit denoted by the dashed line.
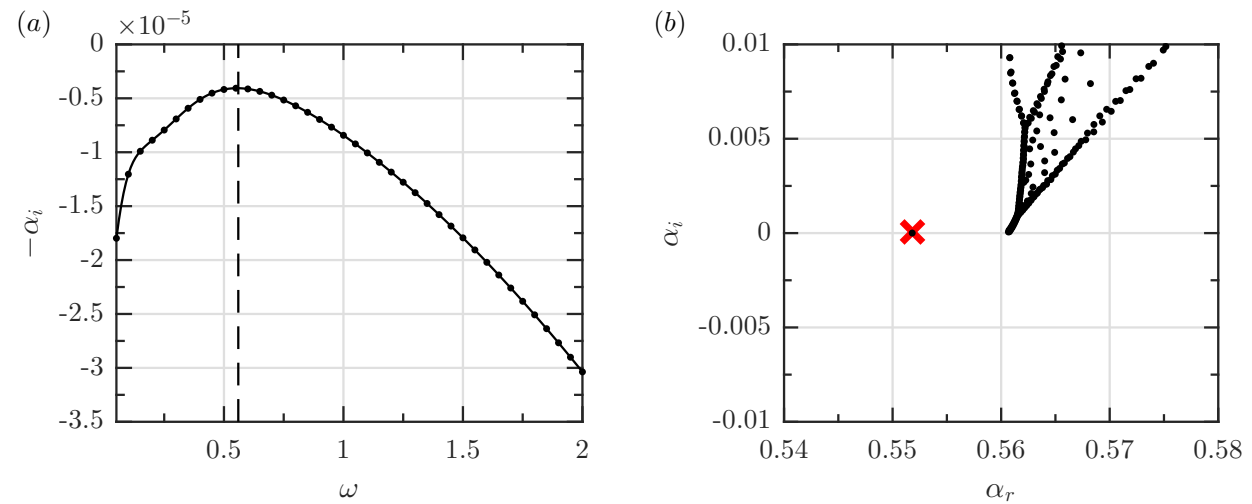

Figure 6: A frequency sweep ( $a$ ) showing the growth rate, $-\alpha_{i}$, as a function of frequency $\omega$ for the fitted Batchelor-vortex parameters. The dashed vertical line indicates the point of maximum growth rate and the corresponding frequency $(\omega=0.56$ ) examined throughout this study. The corresponding eigenvalue spectrum is shown in $(b)$ with the most unstable eigenvalue denoted by the red $\times$.

for vortex wandering; the type of instability, however, is still up for debate. The instability could be the response of stochastic forcing by wind-tunnel turbulence, requiring a frequency response (resolvent) analysis to quantify the amplification. Alternatively, an inherent instability could be at the heart of the energy growth, requiring an initial-value (matrix exponential) analysis to determine further details. In either case, for nearly neutrally stable configurations, as is the case in our study, a robust flow structure, consisting of the neutral stability eigenfunction, is expected. Moreover, we point out that our analysis assumed a parallel base flow: a streamwise variation of the base flow has not been accounted for. For upstream positions, however, the two-dimensional wake component is certainly significant, and a pure Batchelor-vortex base flow profile will show its limitations. 
(a)

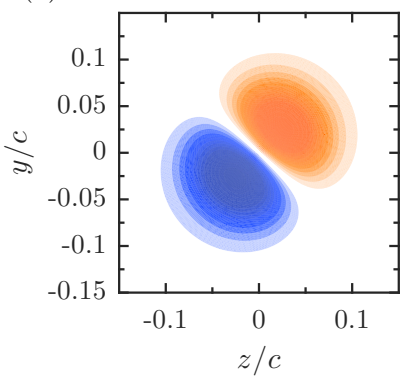

(d)

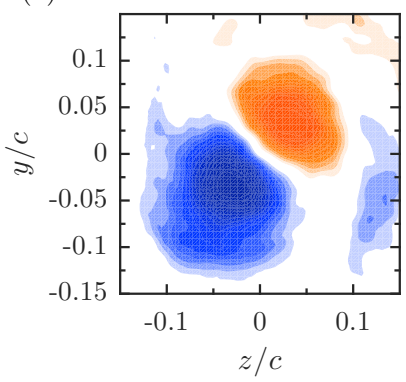

(b)

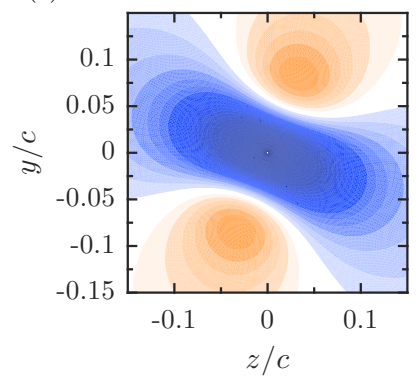

$(e)$

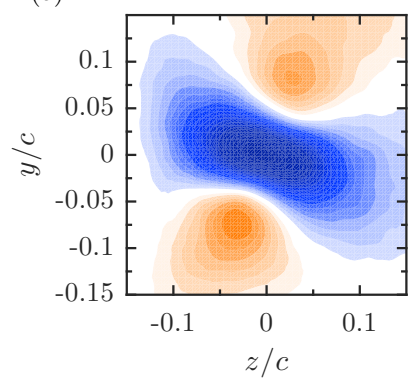

(c)

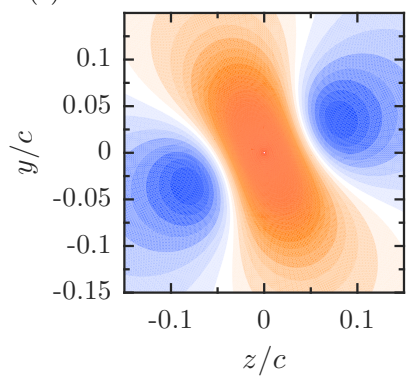

$(f)$

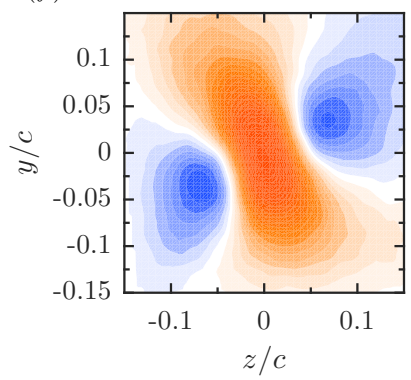

Figure 7: Eigenfunctions and POD modes of the $\tilde{u}, \tilde{v}$, and $\tilde{w}$ velocities corresponding to the left, middle, and right columns, respectively. Figures $(a)-(c)$ show the eigenfunctions of the most unstable modes from the spatial stability analysis of the Batchelor vortex. Figures $(d)-(f)$ show the principal POD mode of the coherent wandering motion from experiments at $x / c=5$.

\section{Conclusions}

Using experimental measurements and a spatial stability analysis of a trailing vortex, we argue that the cause of vortex wandering is a vortex instability rather than being solely a result of wind tunnel effects. By correcting for the wandering measured in stereoscopic particle image velocimetry (SPIV) data, we extract the wandering motion via a triple decomposition and perform proper orthogonal decomposition (POD) to examine the most energetic POD mode, which is helical in nature, $(|m|=1)$. We first examine the formation of this mode as it progresses downstream, showing that a two-lobed structure is developed beyond $x / c=1.5$. Furthermore, the two-dimensional wake is coupled to the vortex dynamics upstream of $x / c=1.5$, indicating that, in the vortex near-field, the wake is significant. On the centerline, the transverse velocities of the identified modes displace the vortex in a wandering manner. These modes diffuse downstream, while their coherent kinetic energy grows - further corroborating our proposition of an underlying instability.

We performed a spatial stability analysis on a matched Batchelor vortex, which produced a primary instability mode identical, in size and structure, to the experimentally identified POD mode. The frequency of the wandering motion is on the order of $\mathcal{O}(1-10 \mathrm{~Hz})$, which closely matches the frequency range reported in previous studies (Devenport et al. 1996; Bailey \& Tavoularis 2008). The growth rate from the stability analysis, however, shows marginal stability, which does not allow us to uniquely identify the type of amplification mechanism (inherent instability or selective noise 
amplification). Despite this shortcoming, the structure of the associated dominant mode (from both stability and POD-analyses) could be identified in a robust manner, and an underlying selection mechanism based on a vortex instability can be safely proposed.

The presence of a non-zero transverse velocity on the centerline despite the presence of the wake indicates that vortex wandering is decoupled from the two-dimensional wake. This implies that the wandering is present despite wind-tunnel effects, and correcting for wandering is equivalent to removing the effects of the vortex instability. Furthermore, the presence of the two-dimensional wake and vortex in the POD mode suggests that the dynamics of these two components are coupled, showing that both the wake and the vortex must be properly modeled or computed to more accurately capture the near-field trailing vortex dynamics.

\section{Acknowledgements}

The authors would like to acknowledge Prof. Sean Bailey for stimulating discussions and for sharing their experimental data. Further, Kyle Pascioni and Robert Reger were instrumental in acquiring the experimental data. Financial support for this work was provided by the National Science Foundation (PIRE, grant NSF-0968313) and the Office of Naval Research (grants N00014-10-1-0832 and N00014-15-1-2403), monitored by Dr. Ronald Joslin.

\section{REFERENCES}

Bailey, S.C.C. \& Tavoularis, S. 2008 Measurements of the velocity field of a wing-tip vortex, wandering in grid turbulence. J. Fluid Mech. 601, 281-315.

Baker, G.R., Barker, S.J., Bofah, K.K. \& SAffman, P.G. 1974 Laser anemometer measurements of trailing vortices in water. J. Fluid Mech. 65 (2), 325-336.

Batchelor, G.K. 1964 Axial flow in trailing line vortices. J. Fluid Mech. 20 (4), 645-658.

Beresh, S.J., Henfling, J.F. \& Spillers, R.W. 2010 Meander of a fin trailing vortex and the origin of its turbulence. Exp. Fluids 49 (3), 599-611.

Corsiglia, V.R., Schwind, R.G. \& Chigier, N.A. 1973 Rapid scanning, three-dimensional hot-wire anemometer surveys of wing-tip vortices. J. Aircraft 10 (12), 752-757.

Deem, E., Edstrand, A., Reger, R., Pascioni, K. \& Cattafesta, L. 2013 Deconvolution correction for wandering in wingtip vortex flowfield data. J. Fluid Sci. Techn. 8 (2), 219232.

Devenport, W.J., Rife, M.C., Liapis, S.I. \& Follin, G.J. 1996 The structure and development of a wing-tip vortex. J. Fluid Mech. 312, 67-106.

FABre, David \& JaCQUin, LAUREnt 2004 Viscous instabilities in trailing vortices at large swirl numbers.

Feys, J. \& Maslowe, S.A. 2014 Linear stability of the Moore-Saffman model for a trailing wingtip vortex. Phys. Fluids 26 (2), 024108.

Fontane, J., Brancher, P. \& Fabre, D. 2008 Stochastic forcing of the lamb-oseen vortex. J. Fluid Mech. 613, 233-254.

Hein, S. \& Theofilis, V. 2004 On instability characteristics of isolated vortices and models of trailing-vortex systems. Computers and Fluids 33 (5-6), 741-753.

Heyes, A.L., Jones, R.F. \& Smith, D.A.R. 2004 Wandering of wing-tip vortices. In 12th Int. Symp. on Appl. of Laser Techn. to Fluid Mech., pp. 1-20. Lisbon, Portugal.

Jacquin, L., Fabre, D., Geffroy, P. \& Coustols, E. 2001 The properties of a transport aircraft wake in the extended near field: an experimental study. In Aerosp. Sci. Meeting, pp. 2001-1038.

Mathew, J., Bahr, C., Sheplak, M., Carroll, B. \& Cattafesta, L. 2005 Characterization of an anechoic wind tunnel facility. In ASME 2005 Int. Mech. Eng. Congr. and Expo., pp. 281-285. Univ. of Florida. 
Mohseni, Kamran \& Colonius, Tim 2000 Numerical Treatment of Polar Coordinate Singularities. Journal of Computational Physics 157 (2), 787-795.

Moore, D.W. \& Saffman, P.G. 1973 Axial flow in laminar trailing vortices. Proc. Roy. Soc. Lond. 333, 491-509.

Olendraru, C \& Sellier, A 2002 Viscous effects in the absolute-convective instability of the Batchelor vortex. Journal of Fluid Mechanics 459, 371-396.

PARedes, P. 2014 Advances in global instability computations: from incompressible to hypersonic flows. PhD thesis, Univ. Politécnica de Madrid.

del Pino, C., Parras, L., Felli, M. \& Fernandez-Feria, R. 2011 Structure of trailing vortices: comparison between particle image velocimetry measurements and theoretical models. Phys. Fluids 23 (1), 013602-13.

Reynolds, W.C. \& Hussain, A.K.M.F. 1972 The mechanics of an organized wave in turbulent shear flow. Part 3. Theoretical models and comparisons with experiments. J. Fluid Mech. 54 (2), 263-288.

Rokhsaz, K., Foster, S.R. \& Miller, L.S. 2000 Exploratory study of aircraft wake vortex filaments in a water tunnel. J. Aircraft 37 (6), 1022-1027.

Sirovich, L. 1987 Turbulence and the dynamics of coherent structures. Part 1: Coherent structures. Q. Appl. Math. 45 (3), 561-571. 